\title{
Safety Tracking Document
}

National Cancer Institute

\section{Source}

National Cancer Institute. Safety Tracking Document. NCI Thesaurus. Code C115728.

Records used to track activities related to safety reporting during the course of a clinical trial. 